\title{
Article \\ Overall Survival and Prognostic Factors among Older Patients with Metastatic Pancreatic Cancer: A Retrospective Analysis Using a Hospital Database
}

\author{
Catherine Conti ${ }^{1}$, Frédéric Pamoukdjian ${ }^{2,3, * \mathbb{D}}$, Thomas Aparicio ${ }^{4} \mathbb{D}$, Soraya Mebarki ${ }^{1}$, Johanne Poisson ${ }^{1,4}$, \\ Gilles Manceau ${ }^{5}$ (D), Julien Taieb ${ }^{6}$, Bastien Rance ${ }^{7,8}$, Sandrine Katsahian ${ }^{9,10}$, Anaïs Charles-Nelson ${ }^{9,10}$ and \\ Elena Paillaud 1,11,*(D)
}

Citation: Conti, C.; Pamoukdjian, F.; Aparicio, T.; Mebarki, S.; Poisson, J.;

Manceau, G.; Taieb, J.; Rance, B.;

Katsahian, S.; Charles-Nelson, A.; et al. Overall Survival and Prognostic Factors among Older Patients with Metastatic Pancreatic Cancer: A Retrospective Analysis Using a Hospital Database. Cancers 2022, 14, 1105. https://doi.org/10.3390/ cancers14051105

Academic Editor: Daniel S. Sitar

Received: 26 January 2022

Accepted: 18 February 2022

Published: 22 February 2022

Publisher's Note: MDPI stays neutral with regard to jurisdictional claims in published maps and institutional affiliations.

Copyright: (C) 2022 by the authors. Licensee MDPI, Basel, Switzerland. This article is an open access article distributed under the terms and conditions of the Creative Commons Attribution (CC BY) license (https:// creativecommons.org/licenses/by/ $4.0 /)$.
1 Service de Gériatrie, Hôpital Européen Georges Pompidou, Paris Cancer Institute CARPEM, APHP, 75015 Paris, France; catherine.conti@aphp.fr (C.C.); soraya.mebarki@aphp.fr (S.M.); johanne.poisson@aphp.fr (J.P.)

2 Service de Médecine Gériatrique, Hôpital Avicenne, APHP, 93000 Bobigny, France

3 Inserm UMR_S942, Cardiovascular Markers in Stressed Conditions, MASCOT, Université Sorbonne Paris Nord, 93000 Bobigny, France

4 Service de Gastroentérologie, Hôpital Saint Louis, APHP, Université de Paris, 75010 Paris, France; thomas.aparicio@aphp.fr

5 Service de Chirurgie Digestive et Oncologique, Hôpital Européen Georges Pompidou, Paris Cancer Institute CARPEM, University of Paris, APHP, 75015 Paris, France; gilles.manceau@aphp.fr

6 Service d'Hépatogastroentérologie, Hôpital Européen Georges Pompidou, Paris Cancer Institute CARPEM, Université de Paris, APHP, 75015 Paris, France; julien.taieb@aphp.fr

7 Département d'Informatique Médicale, Hôpital Européen Georges Pompidou, Paris Cancer Institute CARPEM, Université de Paris, APHP, 75015 Paris, France; bastien.rance@aphp.fr

8 INSERM UMR_S 1138 équipe 22, Centre de Recherche des Cordeliers, 75006 Paris, France

9 Unité de Recherche Clinique, Hôpital Européen Georges Pompidou, APHP, 75015 Paris, France; sandrine.kastahian@aphp.fr (S.K.); anais.charles-nelson@aphp.fr (A.C.-N.)

10 INSERM, Centre d'Investigation Clinique 1418 (CIC1418) Épidémiologie Clinique, 75908 Paris, France

11 Clinical, Epidemiology and Ageing, IMRB-UPEC/Inserm U955, Université Paris-Est Creteil, 94000 Creteil, France

* Correspondence: frederic.pamoukdjian@aphp.fr (F.P.); elena.paillaud@aphp.fr (E.P.)

Simple Summary: The benefits of standard treatments in metastatic pancreatic cancer $(\mathrm{mPC})$ in terms of overall survival (OS) remain to date unclear, especially after 70 years. Alongside geriatric and oncologic parameters, we showed that the gemcitabine + nab-paclitaxel regimen and anti-diabetic therapy were significantly associated with a better OS, while impaired functional status, the liver metastases and high neutrophil count were associated with a worse OS in older adults with mPC. We confirm the feasibility and efficacy of chemotherapy in older adults with mPC.

\begin{abstract}
Pre-therapeutic factors associated with overall survival (OS) among older patients $\geq 70$ years with metastatic pancreatic cancer ( $\mathrm{mPC}$ ) are not known. This was a retrospective single-centre cohort study in Paris including 159 consecutive older patients with $\mathrm{mPC}$ between 2000 and 2018. Alongside geriatric parameters, specific comorbidities, cancer-related data and chemotherapy regimens were retrieved. Cox multivariate models were run to assess predictors for OS. The median age was 80 years, $52 \%$ were women, $21.5 \%$ had diabetes, and $48 \%$ had pancreatic head cancer and $72 \%$ liver metastases. $62 \%$ of the patients $(n=99)$ received chemotherapy, among which the gemcitabine + nab-paclitaxel $(\mathrm{GnP})$ regimen was the most frequent $(72 \%)$. Median OS $[95 \% \mathrm{CI}]$ was $7.40[5.60-10.0]$ and 1.40 [0.90-2.20] months respectively for patients with and without chemotherapy. The GnP regimen (aHR $[95 \% \mathrm{CI}]=0.47[0.25-0.89], p=0.02)$ and diabetes $(\mathrm{aHR}=0.44[0.24-0.77], p=0.004)$ (or anti-diabetic therapy) were multivariate protective factors for death, while ECOG-PS, liver metastases, and the neutrophil cell count were multivariate risk factors for death. In the chemotherapy group, ECOGPS, number of metastatic sites and the GnP remained significantly associated with OS. Our study confirms the feasibility and efficacy of chemotherapy and the protective effects of diabetes among older patients with $\mathrm{mPC}$.
\end{abstract}


Keywords: pancreatic cancer; chemotherapy; older adults; overall survival; prognostic factors; diabetes; functional status

\section{Introduction}

With a median age of 69 years and 73 years at diagnosis for men and women respectively, pancreatic cancer is a disease of the elderly. Despite advances in cancer treatment, pancreatic cancer, mainly diagnosed at a metastatic stage, remains one of the solid cancers worldwide with the poorest diagnosis, and its incidence and mortality increase with age [1].

The standard treatment for metastatic pancreatic cancer $(\mathrm{mPC})$ has for a long time been gemcitabine alone [2]. In 2011, compared to gemcitabine alone, the PRODIGE-4/ACCORD11 clinical trial showed the superiority of the FOLFIRINOX regimen (combining 5- fluorouracil + leucovorin + oxaliplatin + irinotecan) as first-line treatment for overall survival in $\mathrm{mPC}$ (11.1 months vs. 6.8 months; $\mathrm{HR}=0.57, p<0.001$ ) [3]. In addition, compared to gemcitabine alone, the MPACT clinical trial [4] in 2013 showed the superiority of a regimen combining gemcitabine + nab-paclitaxel $(\mathrm{GnP})$ first line for overall survival $(8.5$ months vs. 6.7 months; $\mathrm{HR}=0.72, p<0.001$ ). These two regimens are now considered the standard care for $\mathrm{mPC}$. However, older adults $\geq 70$ years were not represented in these two trials.

While some older patients could benefit from chemotherapy, the benefits of standard treatments in $\mathrm{mPC}$ in terms of survival remain to date unclear, especially after 70 years $[5,6]$. Indeed, regarding elderly patients, the main concern is patient heterogeneity in terms of comorbidities, dependency, malnutrition or functional status, each of these factors being liable to be associated with poor outcomes in the course of cancer treatment [7]. To clarify this point, the Geriatric Assessment (GA), which is a multidimensional health assessment for older adults, was initially recommended to guide cancer-treatment decisions [8], but no study to date has assessed the predictive value of the GA for overall survival among older patients with $\mathrm{mPC}$.

The identification of pre-therapeutic factors taking into account geriatric and oncologic parameters that are associated with shorter survival could help select older patients who could benefit from standard chemotherapy.

Here, we aimed to assess pre-therapeutic factors that were associated with overall survival during first-line treatment among older adults $\geq 70$ years with $\mathrm{mPC}$, and to compare patients who received chemotherapy with those who did not.

\section{Materials and Methods}

In this study, we followed the recommendations of the STrengthening the Reporting of OBservational studies in Epidemiology method (STROBE) for the reporting of observational epidemiological studies [9].

\subsection{Study Design and Population}

A retrospective analysis of older patients with $\mathrm{mPC}$ and treated between 1 October 2000 and 31 October 2018 in a single centre in Paris (Georges Pompidou European Hospital) was performed. All consecutive older adults $\geq 70$ years with histologically confirmed primitive pancreatic cancer at metastatic stage at diagnosis and with no previous treatment were included. Neuroendocrine tumors and pancreatic lymphoma were excluded.

All consecutive eligible patients were retrieved from medical records using the International Classification of Diseases, v10, if they were coded as follows: "malignant neoplasm of the pancreas" (C25) + "secondary malignant neoplasm of the liver and intrahepatic bile duct" (C78.7) + "secondary malignant neoplasm in unspecified location "(C79.9) [10].

The inclusion date was the date of the diagnosis.

The study was approved by the local ethics committee (CERAPHP; reference: 10 July 2021). 


\subsection{Data Collection}

At the time of the first oncology consultation, demographic data including age, gender, and marital status (married/single) was recorded.

Cancer-related data was collected in the course of the first line of treatment: date of diagnosis, location (tail, body, isthmus, head), number of metastatic sites, Eastern Cooperative Oncology Group Performance Status (ECOG-PS), and presence of ascites at diagnosis or endobiliary stenting. The number of metastatic sites was determined using the CT-scan reports or medical records or during weekly multidisciplinary meeting reports. Cancer treatment was classified as follows: single gemcitabine or gemcitabine + nab-paclitaxel $(\mathrm{GnP})$ or 5-fluorouracil/leucovorin + oxaliplatin (FOLFOX) or fluorouracil/leucovorin + irinotecan + oxaliplatin (FOLFIRINOX) or 5-fluorouracil simplified (5-FU) or capecitabine alone or exclusively supportive care. Among the chemotherapy regimens, single gemcitabine was chosen as the reference.

Geriatric parameters were retrospectively retrieved from medical records and included the following 7 domains: total comorbidities (Charlson's updated comorbidity index $\geq 1$ ) and specific comorbidities (diabetes, heart failure, renal failure, chronic respiratory failure, liver failure, history of stroke); poly-medication ( $\geq 5$ drugs a day) [11]; dependency (Activity of Daily Living scale (ADL) $\leq 5 / 6$ ) [12]; walking limitations (walks alone or with help) [13]; malnutrition (weight loss $\geq 5 \%$ in the previous year; body mass index (BMI) $<21 \mathrm{~kg} / \mathrm{m}^{2}$; albumin level < $35 \mathrm{~g} / \mathrm{L}$ ) [14,15]; and history of depressed mood or cognitive impairment.

Covariates were as follows: neutrophil cell count $(\mathrm{G} / \mathrm{L})$, lymphocyte cell count $(\mathrm{G} / \mathrm{L})$, neutrophil to lymphocyte ratio (NLR), haemoglobin level (anaemia defined as below 12 g/dL); C-Reactive Protein (CRP) (mg/L), Ca-19.9 (kUI/L), and total bilirubin level $(\mu \mathrm{mol} / \mathrm{L})$. We also considered type of diabetes treatment as covariates as follows: no treatment, insulin alone, oral anti-diabetic drugs or insulin + oral anti-diabetic drugs.

\subsection{Outcomes}

The primary outcome was overall survival (OS). Vital status was determined from the medical records or the public records office.

The secondary outcome was the profile of patients with chemotherapy.

\subsection{Statistical Analysis}

Categorical variables were described as numbers (\%) and quantitative variables were described as a means $\pm \mathrm{SD}$ or median (min-max), as appropriate.

Categorical variables were compared with Pearson's chi-square test or Fisher's exact test, as appropriate. Continuous variables were compared using Student's $t$ test or Wilcoxon's test, as appropriate.

Overall survival: Univariate survival curves were estimated according to the KaplanMeier method for chemotherapy status (yes/no) in the whole cohort, then for number of metastasis sites ( $\leq 1$ vs. $>1$ ), for ECOG-PS (0-2 vs. 3-4), and for chemotherapy regimens (by reference for single gemcitabine) in the chemotherapy subgroup. Cox uni- and multivariate proportional hazards regression models were run to assess pre-therapeutic factors associated with overall survival. The model assumptions were verified. Variables yielding $p$-values (Wald test) under 0.20 and with less than $15 \%$ of missing data in the univariate analysis were considered for inclusion in the multivariate analysis. A stepwise selection process based on the lowest Akaike criterion was performed to retain the final multivariate models. Additional stratified analyses according to chemotherapy status (yes/no) were also performed. The association between pre-therapeutic factors and overall survival was expressed using adjusted hazard ratios (aHRs) and a 95\% CI.

All tests were two-sided, and the threshold for statistical significance was set at $p<0.05$. The data was analysed using $R$ statistical software (version4.0.2, R Foundation for Statistical Computing, Vienna, Austria; http:/ / www.r-project.org, accessed on 10 January 2022). 


\section{Results}

\subsection{Patients}

During the study period, 159 consecutive patients aged 70 and over with $\mathrm{mPC}$ were retrospectively included in this study (Figure 1).

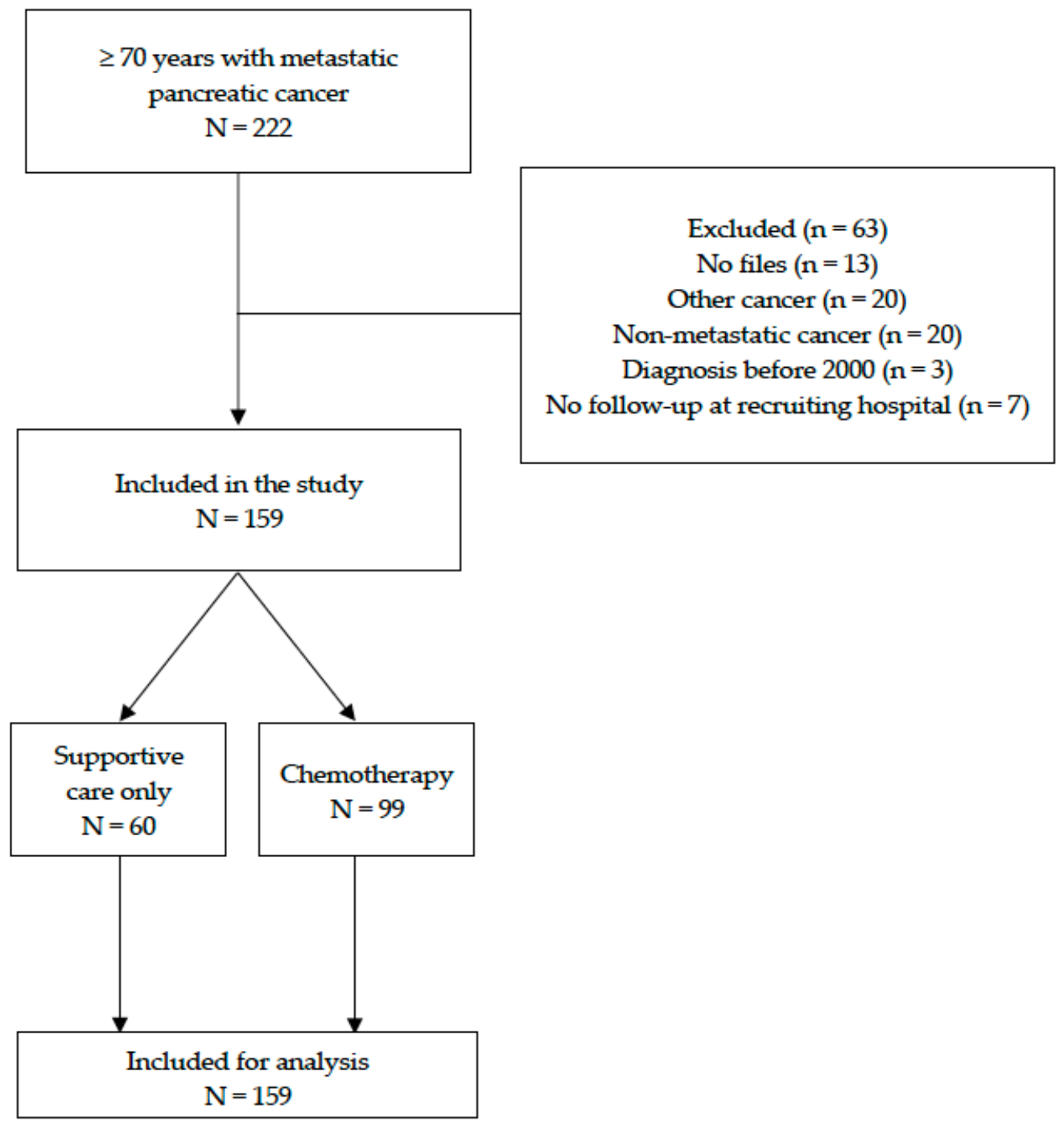

Figure 1. Flow chart for the selection process.

\subsection{Baseline Characteristics and Comparison between Patients with and without Chemotherapy}

Table 1 shows the baseline characteristics of the 159 patients with mPC. The mean age was $80.2 \pm 6.3$ years. A majority of patients were women (52\%), had pancreatic head cancer $(48 \%)$ mainly with liver metastases $(72 \%)$ and a low ECOG-PS score $(\geq 3)$. According to the tools and thresholds used, the level of impairment in the domains explored by the geriatric parameters varied from $9 \%$ (cognitive impairment) to 66\% (weight loss and albumin $<35 \mathrm{~g} / \mathrm{L})$. Among comorbidities, heart failure $(33.5 \%)$ and diabetes $(21.5 \%)$ were the most frequent. Among anti-diabetic treatments, oral anti-diabetic drugs concerned $19 / 34(56 \%)$ of the diabetic patients. The distribution of oral anti-diabetic drugs was available for 17 of them as follows: 14/17 (82\%) were treated with metformin, $7 / 17(41 \%)$ with glinides, and 4/17 (23.5\%) with sulfonamides. 
Table 1. Baseline characteristics and comparison between patients with chemotherapy and exclusively supportive care among 159 older adults with metastatic pancreatic cancer.

\begin{tabular}{|c|c|c|c|c|}
\hline Variables & Whole Cohort & Chemotherapy & Supportive Care & $p^{*}$ \\
\hline & $n=159(\%)$ & $n=99(\%)$ & $n=60(\%)$ & \\
\hline Age (y), median (min-max) & $80.0(70.0-98.0)$ & $77.0(70.0-93.0)$ & $83.0(70.0-98.0)$ & $<0.001$ \\
\hline Gender (female) & $83(52)$ & $55(56)$ & $28(47)$ & 0.27 \\
\hline \multicolumn{5}{|l|}{ Cancer site $(n=158)$} \\
\hline Head & $76(48)$ & $53(53.5)$ & $23(38)$ & 0.06 \\
\hline Body & $37(23)$ & $25(25)$ & $12(20)$ & 0.44 \\
\hline Tail & $41(26)$ & $22(22)$ & $19(32)$ & 0.18 \\
\hline Unspecified & $4(2.5)$ & $1(1)$ & $3(5)$ & 0.15 \\
\hline \multicolumn{5}{|l|}{ Metastases $(n=153)$} \\
\hline $\mathrm{N}^{\circ}$ of metastasis sites, median (min-max) & $1.0(1.0-4.0)$ & $1.0(1.0-4.0)$ & $1.0(1.0-3.0)$ & 0.9 \\
\hline Lymph nodes & $20(13)$ & $12(12)$ & $8(13)$ & 0.8 \\
\hline Liver & $115(72)$ & $66(67)$ & $49(82)$ & 0.04 \\
\hline Lung & $27(17)$ & $21(21)$ & $6(10)$ & 0.06 \\
\hline Peritoneal carcinomatosis & $43(27)$ & $30(30)$ & $13(22)$ & 0.2 \\
\hline Bone & $9(6)$ & $5(5)$ & $4(7)$ & 0.7 \\
\hline Ascites (yes), $n=145$ & 25 (17) & $13(14)$ & $12(22)$ & 0.22 \\
\hline Endobiliary prosthesis (yes), $n=156$ & $40(26)$ & $28(29)$ & $12(20)$ & 0.24 \\
\hline $\begin{array}{l}\text { Ca-19.9 (KUI/L), median (min-max), } \\
n=116\end{array}$ & $20.0(0.0-71.0)$ & $18.0(0.0-26.5)$ & $77.5(0.0-71.0)$ & 0.11 \\
\hline Marital status (single), $n=130$ & $65(50)$ & $34(42)$ & $31(64)$ & 0.01 \\
\hline Alcohol consumption (yes), $n=123$ & $12(10)$ & $8(11)$ & $4(8)$ & 0.55 \\
\hline Active smoker (yes), $n=123$ & $37(30)$ & $23(32)$ & $14(27)$ & 0.51 \\
\hline ECOG-PS, median (min-max), $n=141$ & $3.0(1.0-5.0)$ & $3.0(1.0-4.0)$ & $3.0(1.0-5.0)$ & $<0.0001$ \\
\hline \multicolumn{5}{|l|}{ Comorbidities $(n=158)$} \\
\hline Charlson's index $\geq 1$ & $80(51)$ & $51(51.5)$ & $29(49)$ & 0.77 \\
\hline Diabetes & $34(21.5)$ & $26(26)$ & $8(14)$ & 0.06 \\
\hline Heart failure & $53(33.5)$ & $30(30)$ & $23(39)$ & 0.26 \\
\hline Kidney failure & $2(1)$ & $0(0)$ & $2(3)$ & 0.14 \\
\hline Respiratory failure & $2(1)$ & $0(0)$ & $2(3)$ & 0.14 \\
\hline Hepatic failure & $0(0)$ & - & - & - \\
\hline Stroke & $8(5)$ & $5(5)$ & $3(5)$ & 1 \\
\hline Poly-medication ( $\geq 5$ drugs a day), $n=155$ & $58(37)$ & $39(40)$ & $19(33)$ & 0.42 \\
\hline ADL-dependency $(\leq 5 / 6), n=136$ & $24(18)$ & $2(2)$ & $22(45)$ & $<0.0001$ \\
\hline Walking limitations (yes), $n=130$ & $21(16)$ & $5(6)$ & $16(33)$ & $<0.0001$ \\
\hline \multicolumn{5}{|l|}{$\begin{array}{l}\text { Malnutrition } \\
\text { Mald }\end{array}$} \\
\hline Weight loss $\geq 5 \%, n=119$ & $79(66)$ & $48(61.5)$ & $31(76)$ & 0.12 \\
\hline $\mathrm{BMI}<21 \mathrm{~kg} / \mathrm{m}^{2}, n=105$ & $38(36)$ & $31(40)$ & $7(26)$ & 0.19 \\
\hline Albumin $<35 \mathrm{~g} / \mathrm{L}, n=107$ & $71(66)$ & $43(59)$ & $28(82)$ & 0.01 \\
\hline CAR, median (min-max), $n=82$ & $1.02(0.00-33.3)$ & $0.80(0.00-33.3)$ & $2.60(0.10-17.2)$ & 0.001 \\
\hline $\begin{array}{l}\text { Neutrophil cell count, median (min-max), } \\
\qquad n=144\end{array}$ & $6.20(1.50-33.3)$ & $5.80(1.5-29.0)$ & $7.70(2.50-33.3)$ & $<0.0001$ \\
\hline NLR, median (min-max), $n=144$ & $5.40(0.30-88.0)$ & $4.40(0.30-88.0)$ & $7.60(1.50-46.5)$ & $<0.0001$ \\
\hline Depression (yes), $n=156$ & $22(14)$ & $15(15)$ & $7(12)$ & 0.62 \\
\hline Cognitive impairment (yes), $n=155$ & $14(9)$ & $6(6)$ & $8(14)$ & 0.09 \\
\hline Haemoglobin $<12 \mathrm{~g} / \mathrm{dL}, n=145$ & $76(52)$ & $43(48)$ & $33(60)$ & 0.15 \\
\hline $\begin{array}{l}\text { Total bilirubin }(\mu \mathrm{mol} / \mathrm{L}) \text {, median } \\
\quad(\min -\max ), n=139\end{array}$ & $16.0(0.00-545)$ & $14.0(0.00-545)$ & $26.5(5.00-466)$ & 0.004 \\
\hline \multicolumn{5}{|l|}{ Anti-diabetic therapy, $n=34$} \\
\hline None & $5(15)$ & $3(11.5)$ & $2(25)$ & \\
\hline Insulin alone & $5(15)$ & $3(11.5)$ & $2(25)$ & 0.37 \\
\hline Oral anti-diabetic drugs alone & $19(56)$ & $15(58)$ & $4(50)$ & \\
\hline Insulin + oral anti-diabetic drugs & $5(15)$ & $5(19)$ & $0(0)$ & \\
\hline \multicolumn{5}{|l|}{ Chemotherapy regimens } \\
\hline Gemcitabine alone & & $49(49)$ & & \\
\hline $\mathrm{GnP}$ & & $23(23)$ & & \\
\hline FOLFOX & & $2(2)$ & - & \\
\hline FOLFIRINOX & & $12(12)$ & & \\
\hline Other ** & & $13(13)$ & & \\
\hline
\end{tabular}

${ }^{*} p$ value for chi2 test or Wilcoxon's test as appropriate; Bold $=$ significant $p$ value at a threshold of $5 \%$; ADL $=$ activities of daily living; $\mathrm{BMI}=$ body mass index; $\mathrm{CAR}=\mathrm{CRP}$ to albumin ratio; ECOG-PS = eastern cooperative oncology group performance status; $\mathrm{NLR}=$ neutrophil to lymphocyte ratio; $\mathrm{GnP}=$ gemcitabine + nab-paclitaxel; FOLFOX = 5-fluorouracil/leucovorin + oxaliplatin; FOLFIRINOX = fluorouracil/leucovorin + irinotecan + oxaliplatin; $* *=5$-FU or capecitabine alone. 
The majority of patients received a chemotherapy regimen $(n=99,62 \%)$, mainly gemcitabine alone or $\mathrm{GnP}(n=72 ; 72 \%)$. Patients with a chemotherapy regimen were significantly younger and more often married, there was a significantly lower proportion of liver metastases, and lower scores for ECOG-PS, ADL-dependency, and walking limitations, and a significantly smaller proportion of haemoglobin levels $<12 \mathrm{~g} / \mathrm{dL}$ (anaemia), and a significantly lower CAR, NLR and total bilirubin values (Table 1).

\subsection{Pre-Therapeutic Factors Associated with Overall Survival among Older Patients with Metastatic Pancreatic Cancer}

Median OS [95\%CI] was 7.40 [5.60-10.0] and 1.40 [0.90-2.20] months respectively among patients with and without chemotherapy (Figure 2). Depending on the chemotherapy regimen, median OS was 4.80 [3.90-7.90], 13.5 [11.6-21.3], 3.65 [2.00-NA], and 10.1 [8.80-NA] for gemcitabine alone, GnP, FOLFOX and FOLFIRINOX, respectively. The median chemotherapy duration was 64 days. Depending on the chemotherapy regimen, the median time of exposure was 38.0, 108.0, 60.5 and 64.5 days for gemcitabine alone, GnP, FOLFOX and FOLFIRINOX, respectively. There were no significant differences between median times of exposure to chemotherapy regimens $(p=0.20)$. For diabetes status, there was no significant difference across the different chemotherapy regimens in terms of median chemotherapy duration $(p=0.90)$.

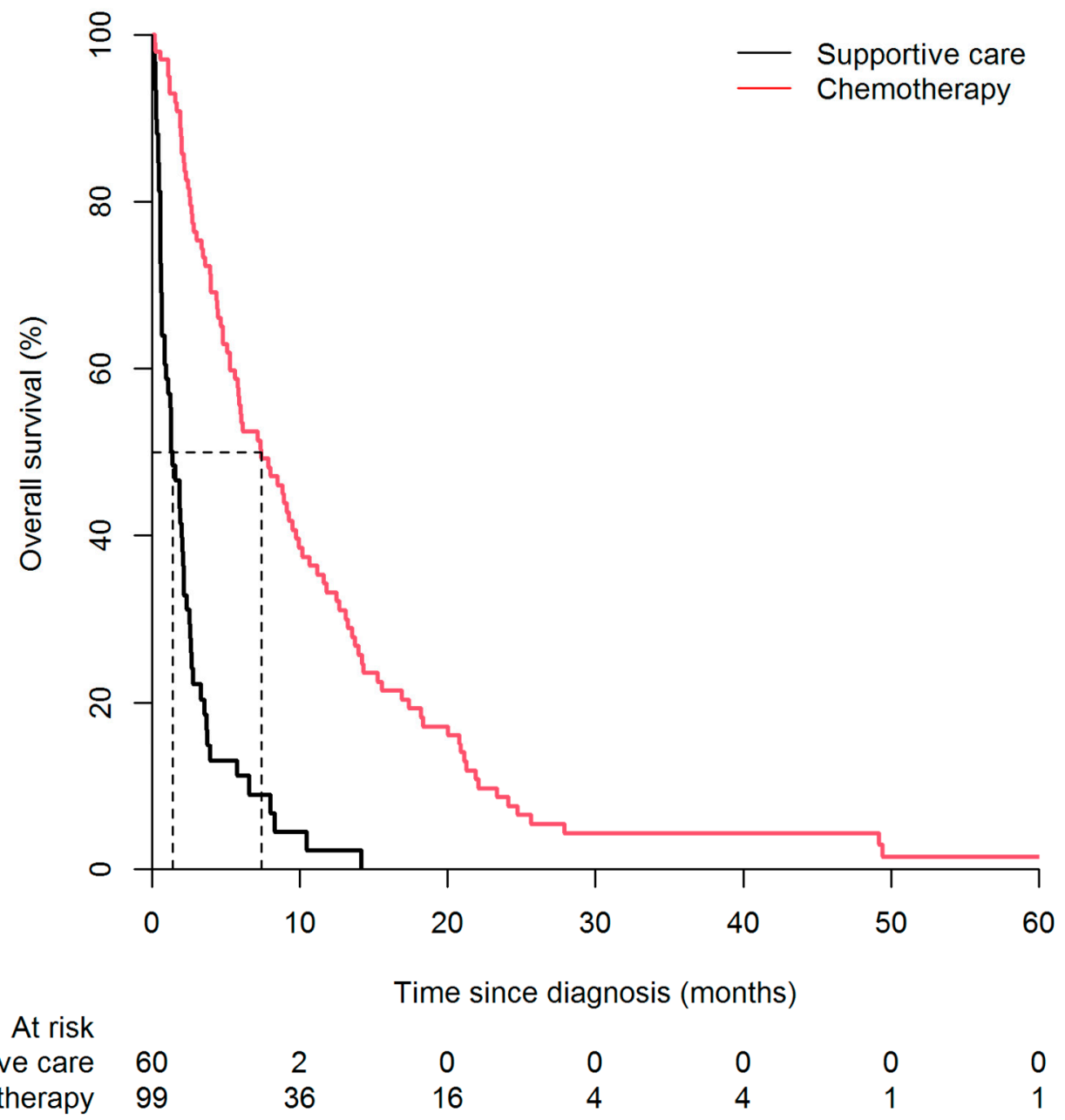

Figure 2. Kaplan-Meier survival curve according to chemotherapy status among older adults with $\mathrm{mPC}$. Dotted lines = median survival in each group.

Whole cohort (Table 2): In univariate analysis, age, ECOG-PS, the ADL scale, walking limitations, diabetes, tumour site, liver metastases, the NLR and the type of chemotherapy 
regimen were significantly associated with overall survival. Due to a collinearity between ECOG-PS and ADL, we retained ECOG-PS for multivariate analyses. Due to a collinearity between diabetes and anti-diabetic therapy, we provided two multivariate models, one including diabetes (model 1), the other including anti-diabetic therapy as covariate (model 2). In both multivariate models, while chemotherapy regimens and diabetes or anti-diabetic therapy were significant protective factors for mortality, the ECOG-PS, liver metastases, and neutrophil cell count (but not the NLR) were significant risk factors for mortality. There was no significant interaction among the multivariate predictors.

Table 2. Factors associated with overall survival in the whole cohort of older adults with mPC.

\begin{tabular}{|c|c|c|c|c|c|c|c|c|c|}
\hline \multirow[t]{2}{*}{ Variables } & \multicolumn{3}{|c|}{ Univariate Analysis } & \multicolumn{3}{|c|}{ Multivariate Model 1} & \multicolumn{3}{|c|}{ Multivariate Model 2} \\
\hline & HR & {$[95 \% \mathrm{CI}]$} & $p^{*}$ & HR & {$[95 \% \mathrm{CI}]$} & $p^{*}$ & HR & {$[95 \% \mathrm{CI}]$} & $p^{*}$ \\
\hline Age & 1.04 & [1.01-1.07] & 0.009 & - & - & - & - & - & - \\
\hline Gender (female) & 0.82 & [0.59-1.13] & 0.21 & & & & & & \\
\hline \multicolumn{10}{|l|}{ Cancer site } \\
\hline Head & 0.55 & {$[0.40-0.77]$} & $<0.0001$ & - & - & - & - & - & - \\
\hline Body & 1.06 & {$[0.72-1.55]$} & 0.77 & & & & & & \\
\hline Tail & 1.55 & {$[1.07-2.26]$} & 0.02 & - & - & - & - & - & - \\
\hline Unspecified & 6.39 & [2.30-17.7] & $<0.0001$ & - & - & - & - & - & - \\
\hline \multicolumn{10}{|l|}{ Metastases } \\
\hline $\mathrm{N}^{\circ}$ of metastasis sites & 1.62 & {$[1.23-2.14]$} & 0.001 & 1.37 & {$[0.90-2.07]$} & 0.13 & 1.44 & {$[0.96-2.12]$} & 0.07 \\
\hline Lymph nodes & 1.05 & {$[0.63-1.74]$} & 0.85 & & & & & & \\
\hline Liver & 1.52 & [1.05-2.18] & 0.02 & 1.71 & [1.06-2.77] & 0.02 & 1.61 & {$[1.00-2.55]$} & 0.04 \\
\hline Lung & 0.81 & {$[0.53-1.23]$} & 0.32 & & & & & & \\
\hline Peritoneal carcinomatosis & 1.12 & {$[0.78-1.61]$} & 0.54 & & & & & & \\
\hline Bone & 1.49 & {$[0.75-2.93]$} & 0.25 & & & & & & \\
\hline Other & 1.6 & [0.88-2.89] & 0.12 & 0.4 & {$[0.13-1.16]$} & 0.09 & 0.39 & {$[0.13-1.15]$} & 0.09 \\
\hline Ascites (yes) & 1.21 & {$[0.77-1.91]$} & 0.4 & & & & & & \\
\hline Endo-biliary prosthesis (yes) & 0.73 & {$[0.50-1.06]$} & 0.09 & - & - & - & - & - & - \\
\hline Ca-19.9 (kUI/L) & 1 & [1.00-1.00] & 0.07 & - & - & - & - & - & - \\
\hline Marital status (alone) & 1.29 & {$[0.90-1.84]$} & 0.16 & - & - & - & - & - & - \\
\hline Alcohol consumption (yes) & 1.34 & {$[0.73-2.44]$} & 0.35 & & & & & & \\
\hline Active smoker (yes) & 0.95 & {$[0.63-1.41]$} & 0.78 & & & & & & \\
\hline ECOG-PS & 1.91 & {$[1.53-2.37]$} & $<0.0001$ & 1.47 & [1.09-1.99] & 0.01 & 1.45 & [1.08-1.93] & 0.01 \\
\hline \multicolumn{10}{|l|}{ Comorbidities } \\
\hline Charlson's index & 1.03 & [0.92-1.15] & 0.59 & & & & & & \\
\hline Diabetes & 0.56 & {$[0.37-0.85]$} & 0.006 & 0.43 & {$[0.24-0.77]$} & 0.004 & & & \\
\hline Heart failure & 1.03 & {$[0.73-1.45]$} & 0.86 & & & & & & \\
\hline Kidney failure & 3.98 & [0.97-16.3] & 0.05 & - & - & - & - & - & - \\
\hline Respiratory failure & 2.19 & {$[0.54-8.90]$} & 0.27 & & & & & & \\
\hline Stroke & 0.82 & [0.38-1.77] & 0.62 & & & & & & \\
\hline $\mathrm{N}^{\circ}$ of medications & 0.99 & [0.94-1.05] & 0.76 & & & & & & \\
\hline ADL-scale & 0.65 & {$[0.56-0.74]$} & $<0.0001$ & - & - & - & - & - & - \\
\hline Walking limitations (yes) & 3.57 & [2.08-6.13] & $<0.0001$ & - & - & - & - & - & - \\
\hline \multicolumn{10}{|l|}{ Malnutrition } \\
\hline Weight loss $\geq 5 \%$ & 1.24 & {$[0.84-1.84]$} & 0.27 & & & & & & \\
\hline $\mathrm{BMI}<21 \mathrm{~kg} / \mathrm{m}^{2}$ & 0.88 & [0.59-1.33] & 0.57 & & & & & & \\
\hline $\mathrm{CAR} \geq 1.02$ & 1.48 & [0.94-2.33] & 0.09 & - & - & - & - & - & - \\
\hline Neutrophil (G/L) & 1.1 & [1.06-1.14] & $<0.0001$ & 1.12 & [1.05-1.20] & 0.001 & 1.12 & [1.04-1.18] & 0.001 \\
\hline NLR, median (min-max) & 1.02 & {$[1.00-1.03]$} & 0.02 & 0.98 & [0.96-1.01] & 0.16 & 0.98 & [0.95-1.01] & 0.16 \\
\hline Depression (yes) & 1.19 & {$[0.74-1.92]$} & 0.47 & & & & & & \\
\hline Cognitive impairment (yes) & 1.56 & {$[0.86-2.84]$} & 0.14 & - & - & - & & & \\
\hline Haemoglobin level (g/dL) & 0.97 & {$[0.87-1.08]$} & 0.52 & & & & & & \\
\hline Total bilirubin ( $\mu \mathrm{mol} / \mathrm{L})$ & 1 & [1.00-1.00] & 0.6 & & & & & & \\
\hline Anti-diabetic therapy (yes) & 0.56 & {$[0.36-0.87]$} & 0.01 & & & & 0.43 & {$[0.23-0.81]$} & 0.009 \\
\hline Chemotherapy (yes) & 0.23 & {$[0.16-0.33]$} & $<0.0001$ & 0.22 & {$[0.12-0.41]$} & $<0.0001$ & 0.23 & {$[0.12-0.41]$} & $<0.0001$ \\
\hline
\end{tabular}

* $p$ value for log-rank test; Bold = significant $p$ value at the threshold of $5 \%$; ADL $=$ activities of daily living $\mathrm{BMI}=$ body mass index; CAR = CRP to albumin ratio; ECOG-PS = eastern cooperative oncology group performance status; NLR = neutrophil to lymphocyte ratio.

Patients with chemotherapy (Table 3): In univariate analysis, ECOG-PS, ADL scores, walking limitations, diabetes, primary tumour site, metastasis site, the number of metastatic sites, and the GnP regimen (by reference for single gemcitabine) were significantly associated with overall survival. Figure 3 shows univariate survival curves according to the 
number of metastasis sites, ECOG-PS, and chemotherapy regimens (by reference for single gemcitabine). Due to a collinearity between diabetes and anti-diabetic therapy, we provided two multivariate models, one including diabetes (model 1), the other including anti-diabetic therapy as covariate (model 2). Regardless the final multivariate model, ECOG-PS, the number of metastatic sites and the GnP regimen (by reference for single gemcitabine) remained significantly associated with overall survival.

Table 3. Factors associated with overall survival in the chemotherapy subgroup of older adults with mPC.

\begin{tabular}{|c|c|c|c|c|c|c|c|c|c|}
\hline \multirow[t]{2}{*}{ Variables } & \multicolumn{3}{|c|}{ Univariate Analysis } & \multicolumn{3}{|c|}{ Multivariate Model 1} & \multicolumn{3}{|c|}{ Multivariate Model 2} \\
\hline & HR & {$[95 \% \mathrm{CI}]$} & $p^{*}$ & HR & {$[95 \% \mathrm{CI}]$} & $p^{*}$ & HR & {$[95 \% \mathrm{CI}]$} & $p^{*}$ \\
\hline Age & 1.01 & {$[0.97-1.04]$} & 0.79 & & & & & & \\
\hline Gender (female) & 0.86 & [0.57-1.30] & 0.48 & & & & & & \\
\hline \multicolumn{10}{|l|}{ Cancer site } \\
\hline Head & 0.59 & {$[0.39-0.89]$} & 0.01 & - & - & - & - & - & - \\
\hline Body & 1.19 & {$[0.75-1.91]$} & 0.45 & & & & & & \\
\hline Tail & 1.66 & [1.02-2.73] & 0.04 & 1.69 & {$[0.92-3.11]$} & 0.09 & 1.67 & {$[0.91-3.03]$} & 0.09 \\
\hline \multicolumn{10}{|l|}{ Metastases } \\
\hline $\mathrm{N}^{\circ}$ of metastasis sites & 2.04 & {$[1.43-2.91]$} & $<0.001$ & 1.86 & {$[1.17-2.95]$} & 0.008 & 1.89 & {$[1.43-2.34]$} & 0.007 \\
\hline Lymph nodes & 0.97 & {$[0.50-1.88]$} & 0.93 & & & & & & \\
\hline Liver & 1.21 & {$[0.78-1.86]$} & 0.39 & & & & & & \\
\hline Lung & 0.91 & [0.56-1.49] & 0.72 & & & & & & \\
\hline Peritoneal carcinomatosis & 1.43 & {$[0.91-2.24]$} & 0.11 & - & - & - & - & - & - \\
\hline Bone & 2 & {$[0.81-4.96]$} & 0.13 & - & - & - & - & - & - \\
\hline Other & 2.4 & [1.15-5.01] & 0.01 & - & - & - & - & - & - \\
\hline Ascites (yes) & 1.02 & [0.55-1.89] & 0.95 & & & & & & \\
\hline Endo-biliary prosthesis (yes) & 0.89 & [0.56-1.39] & 0.6 & & & & & & \\
\hline Ca-19.9 (kUI/L) & 1 & [1.00-1.00] & 0.37 & & & & & & \\
\hline Marital status (single) & 0.98 & {$[0.62-1.56]$} & 0.94 & & & & & & \\
\hline Alcohol consumption (yes) & 1.75 & {$[0.82-3.73]$} & 0.15 & - & - & - & - & - & - \\
\hline Active smoker (yes) & 1.05 & {$[0.63-1.77]$} & 0.84 & & & & & & \\
\hline ECOG-PS & 1.69 & [1.22-2.33] & 0.002 & 1.74 & {$[1.20-2.53]$} & 0.003 & 1.75 & [1.38-2.12] & 0.003 \\
\hline \multicolumn{10}{|l|}{ Comorbidities } \\
\hline Charlson's index & 0.98 & [0.83-1.15] & 0.77 & & & & & & \\
\hline Diabetes & 0.6 & [0.37-0.99] & 0.04 & 0.57 & [0.29-1.16] & 0.12 & & & \\
\hline Heart failure & 0.83 & {$[0.53-1.30]$} & 0.41 & & & & & & \\
\hline Stroke & 0.69 & [0.25-1.89] & 0.47 & & & & & & \\
\hline $\mathrm{N}^{\circ}$ of medications & 0.98 & [0.92-1.06] & 0.65 & & & & & & \\
\hline ADL-scale & 0.4 & [0.19-0.83] & 0.01 & - & - & - & - & - & - \\
\hline Walking limitations (yes) & 6.07 & [2.02-18.2] & 0.001 & - & - & - & - & - & - \\
\hline \multicolumn{10}{|l|}{ Malnutrition } \\
\hline Weight loss $\geq 5 \%$ & 1.05 & {$[0.66-1.68]$} & 0.83 & & & & & & \\
\hline $\mathrm{BMI}<21 \mathrm{~kg} / \mathrm{m}^{2}$ & 1 & [0.62-1.59] & 0.98 & & & & & & \\
\hline $\mathrm{CAR} \geq 1.02$ & 1.41 & {$[0.78-2.54]$} & 0.25 & & & & & & \\
\hline Neutrophil (G/L) & 1.07 & [1.00-1.15] & 0.05 & 1.06 & [0.99-1.13] & 0.12 & 1.05 & [0.99-1.10] & 0.1 \\
\hline NLR, median (min-max) & 1.02 & [1.00-1.05] & 0.1 & - & - & - & - & - & - \\
\hline Depression (yes) & 1.23 & {$[0.68-2.22]$} & 0.5 & & & & & & \\
\hline Cognitive impairment (yes) & 1.17 & {$[0.47-2.90]$} & 0.73 & & & & & & \\
\hline Haemoglobin level (g/dL) & 1.01 & {$[0.87-1.17]$} & 0.89 & & & & & & \\
\hline Total bilirubin $(\mu \mathrm{mol} / \mathrm{L})$ & 1 & {$[1.00-1.00]$} & 0.95 & & & & & & \\
\hline Anti-diabetic therapy (yes) & & & & & & & 0.49 & [0.23-1.03] & 0.06 \\
\hline \multicolumn{10}{|l|}{ Chemotherapy regimens } \\
\hline Single gemcitabine & 1.00 (ref) & - & - & $\begin{array}{l}1.00 \\
\text { (ref) }\end{array}$ & - & - & $\begin{array}{l}1.00 \\
\text { (ref) }\end{array}$ & - & - \\
\hline GnP & 0.54 & {$[0.32-0.91]$} & 0.02 & 0.47 & {$[0.25-0.89]$} & 0.02 & 0.45 & {$[0.25-0.87]$} & 0.01 \\
\hline FOLFOX & 2.53 & {$[0.60-10.6]$} & 0.2 & 3.45 & {$[0.77-15.4]$} & 0.1 & 3.41 & [0.77-15.0] & 0.1 \\
\hline FOLFIRINOX & 0.55 & [0.28-1.08] & 0.08 & 0.97 & [0.44-2.15] & 0.94 & 1.01 & {$[0.46-2.20]$} & 0.96 \\
\hline Other $* *$ & 0.87 & [0.46-1.62] & 0.65 & 0.86 & [0.39-1.91] & 0.71 & 0.86 & [0.39-1.89] & 0.71 \\
\hline
\end{tabular}

* $p$ value for log-rank test; Bold $=$ significant $p$ value at the threshold of $5 \%$; ADL $=$ activities of daily living; $\mathrm{BMI}=$ body mass index; $\mathrm{CAR}=\mathrm{CRP}$ to albumin ratio; ECOG-PS = eastern cooperative oncology group performance status; NLR = neutrophil to lymphocyte ratio; GnP = gemcitabine + nab-paclitaxel; FOLFOX = 5fluorouracil/leucovorin + oxaliplatin; FOLFIRINOX = fluorouracil/leucovorin + irinotecan + oxaliplatin; $* * 5$-FU simplified or capecitabine alone. 
A

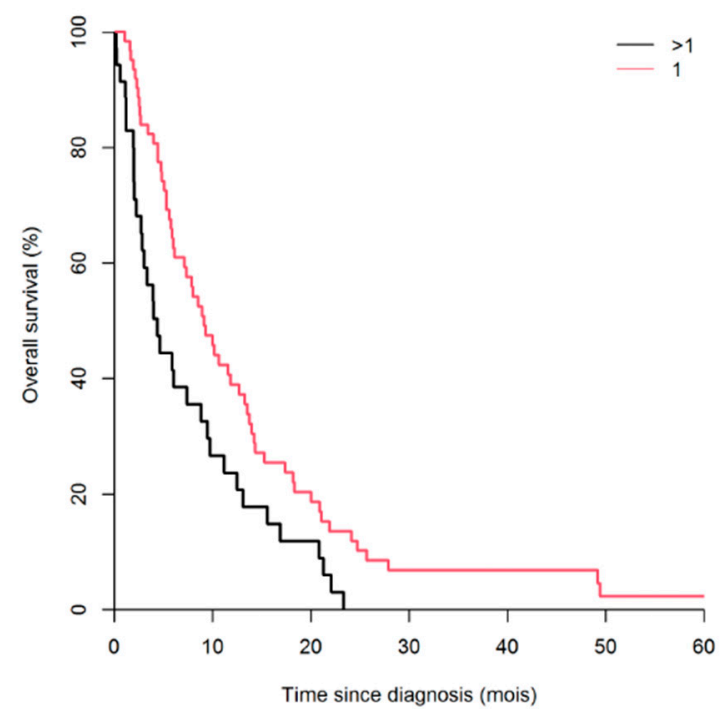

B

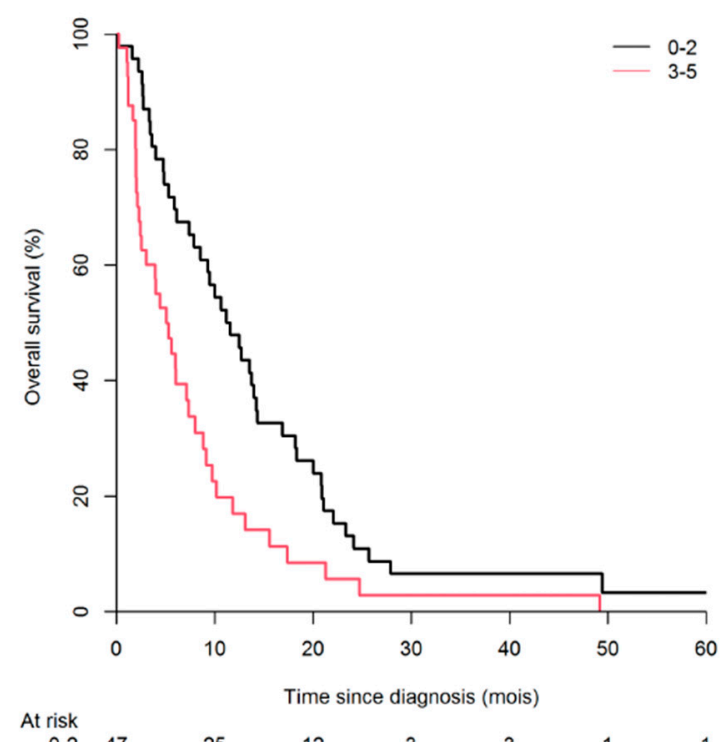

$\mathrm{C}$

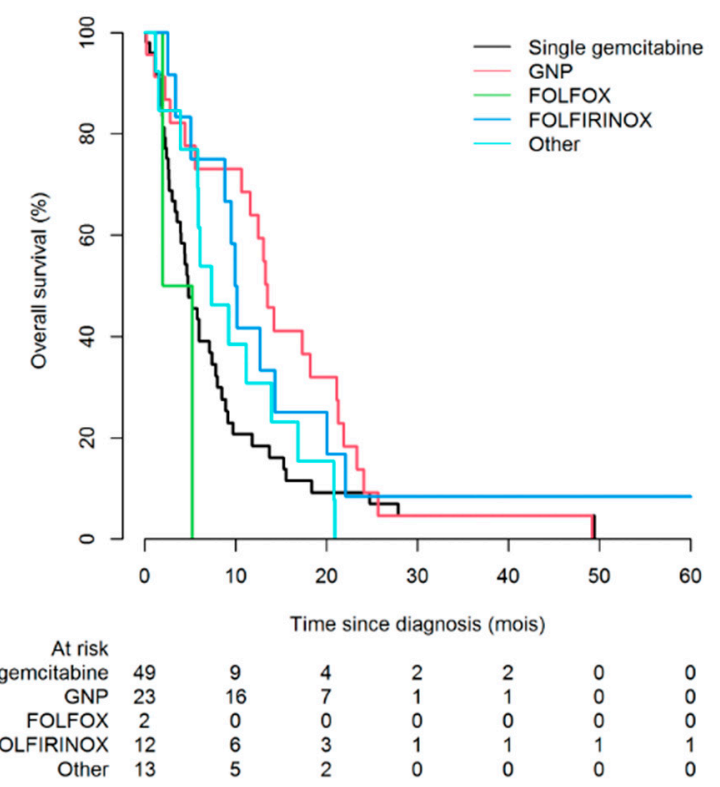

Figure 3. Kaplan-Meier survival curve according to the number of metastatic sites (A), ECOG-PS (B), and chemotherapy regimens (C) among older adults with mPC. 


\section{Discussion}

In this original retrospective study including 159 consecutive older adults with a median age of 80 years and with metastatic pancreatic cancer (mPC) not previously treated, $62 \%$ received chemotherapy, mainly gemcitabine alone or the $\mathrm{GnP}$ regimen. Alongside the geriatric parameters, we found that dependency (ADL and ECOG-PS), liver metastasis, high neutrophil cell count and diabetes (or anti-diabetic therapy) were independent pre-therapeutic factors associated with overall survival (OS). In a stratified analysis of patients with chemotherapy, ECOG-PS, the number of metastatic sites and the GnP regimen remained significantly associated with OS.

The main finding of our study is that pre-therapeutic diabetes and anti-diabetic therapy were significantly associated with better OS among older adults $\geq 70$ years with $\mathrm{mPC}$ in the overall cohort but not in the chemotherapy subgroup (probably because of a lack of power). Although we did not distinguish between type- 1 and type-2 diabetes, because of the retrospective study design, this result could be explained by the significantly lower prevalence of sarcopenia among type- 2 diabetic patients treated with metformin, which was recently highlighted in a large meta-analysis involving 16,800 patients [16]. Indeed, in our study, $82 \%$ of the diabetic patients were treated with metformin which is in line with the literature $[17,18]$. The age-related loss of skeletal muscle mass and function (sarcopenia) is a well-known independent risk factor for shorter survival among cancer patients [19]. The role of metformin combines both anti-cancer activity and protein synthesis via direct and indirect pathways [20]. Metformin was also found to be associated with a significant benefit for overall survival in a large meta-analysis involving 24,178 cancer patients, leading the authors to recommend the use of metformin as an adjuvant treatment for cancer [21]. More recently, the protective effect of metformin on mortality among pancreatic cancer patients was specifically confirmed in a large meta-analysis involving 38,772 patients with a pooled Hazard Ratio of 0.81 [95\%CI: 0.70-0.91]. Nevertheless, in line with our study, this protective effect was not found in the chemotherapy subgroup, with a pooled HR of 0.99 [95\%CI: 0.67-1.30] [22]. Other anti-diabetic drugs including insulin could also have a benefit on muscle mass and function, but there have been no studies to date supporting this hypothesis among older adults, particularly on account of the supposed effect of age-related insulin resistance [23].

Another strength of our study was that for the first time we reported the predictive value of geriatric parameters among older patients with $\mathrm{mPC}$. Indeed, as previously reported in other cancers, we were able to confirm that ADL-dependency and walking limitations were geriatric parameters that were significantly associated with shorter survival during the first line of treatment for $\mathrm{MPC}[7,24]$. We were also able to provide a comprehensive characterization of the geriatric health status in this specific population and before cancer-treatment decisions, which was consistent with other large observational studies in geriatric oncology $[7,25]$. Moreover, in agreement with previous studies, we found that the neutrophil count and the number of metastatic sites were independently associated with OS [26,27].

Regarding the benefit of chemotherapy on overall survival among older patients with $\mathrm{mPC}$, our study results are consistent with other studies on younger patients since we found similar median survival rates associated with chemotherapy regimens [3,4]. Specifically, we found that the use of a chemotherapy regimen in older patients with metastatic pancreatic cancer, added a significant benefit in terms of overall survival, even after adjustment for functional and nutritional status. The best regimen that we found in terms of feasibility and efficacy remains the $\mathrm{GnP}$ regimen (median $\mathrm{OS}=13.5$ months), but the small number of patients probably underestimated the impact of other poly-chemotherapy regimens. Indeed, recent retrospective studies have reported the feasibility and efficacy of the main regimens among older adults with MPC: Pignon et al. reported a median OS of 8.0 months for the $\mathrm{GnP}$ regimen with no significant differences between younger and older patients ( $<$ or $\geq 75$ years) [28]; Mizrahi et al. reported a median OS of 12.2 months for the modified FOLFIRINOX regimen among older adults $\geq 75$ years with $\mathrm{mPC}$ [29]; Berger et al. reported 
a median OS of 10.2 months for the FOLFIRINOX regimen with no significant differences between younger and older adults ( $<$ or $\geq 65$ years) [30]; and Costa et al. reported median OS ranging from 6.7 months (gemcitabine alone) to 13.8 months (FOLFIRINOX) among older adults $\geq 65$ years with mPC [31]. Finally, we confirmed the lesser efficacy of the gemcitabine alone regimen among older adults with $\mathrm{MPC}$. However, our study results should be taken with caution since, due to the retrospective design of our study, the dose-intensity related feasibility of each regimen was not known [32].

The main limitation of our study was the retrospective design with a single centre, which could have led to a patient selection bias, but the consecutive inclusion aimed to reduce this bias. In addition, we considered several clinical and biological parameters to reduce confounding biases. However, further prospective studies are needed to validate our study results using a standardized geriatric assessment [33].

On the basis of our study results, we support the use of chemotherapy, mainly the GnP regimen, among older adults with $\mathrm{mPC}$. Conversely, patients with dependency or walking limitations would probably benefit more from exclusively supportive care, including nutritional and functional rehabilitation. This strategy could be put into perspective with the simple GRADE score (based on weight loss, gait speed, cancer site and cancer extension) which we recently published to help in cancer-treatment decisions concerning older adults and to limit situations of over- or under-treatment [34].

\section{Conclusions}

While the $\mathrm{GnP}$ regimen and anti-diabetic drugs were significant protective factors for death, poor functional status and the number of metastatic sites were significant risk factors for death among older adults with metastatic pancreatic cancer.

Author Contributions: Conceptualization, S.M. and E.P.; methodology, A.C.-N. and S.K.; software, A.C.-N.; validation, C.C., S.M. and A.C.-N., formal analysis, F.P. and A.C.-N.; investigation, C.C. and B.R.; data procurement, C.C. and B.R.; original draft preparation, C.C., F.P. and E.P.; review and editing, T.A., S.M., J.P., G.M., J.T. and S.K.; visualization, C.C. and A.C.-N.; supervision, E.P. All authors have read and agreed to the published version of the manuscript.

Funding: This research received no external funding.

Institutional Review Board Statement: The study was approved by the local ethics committee (CERAPHP; reference: 10 July 2021).

Informed Consent Statement: Informed consent was obtained from all subjects involved in the study.

Data Availability Statement: The data presented in this study are available on request from the corresponding author.

Acknowledgments: We thank Angela Swaine and Sarah Leyshon for revising the manuscript.

Conflicts of Interest: The authors declare no conflict of interest.

\section{References}

1. Huang, J.; Lok, V.; Ngai, C.H.; Zhang, L.; Yuan, J.; Lao, X.Q.; Ng, K.; Chong, C.; Zheng, Z.-J.; Wong, M.C.S. Worldwide Burden of, Risk Factors for, and Trends in Pancreatic Cancer. Gastroenterology 2021, 160, 744-754. [CrossRef] [PubMed]

2. Burris, H.A., III; Moore, M.J.; Andersen, J.; Green, M.R.; Rothenberg, M.L.; Modiano, M.R.; Cripps, M.C.; Portenoy, R.K.; Storniolo, A.M.; Tarassoff, P.; et al. Improvements in Survival and Clinical Benefit with Gemcitabine as First-Line Therapy for Patients with Advanced Pancreas Cancer: A Randomized Trial. J. Clin. Oncol. 1997, 15, 2403-2413. [CrossRef] [PubMed]

3. Conroy, T.; Desseigne, F.; Ychou, M.; Bouché, O.; Guimbaud, R.; Bécouarn, Y.; Adenis, A.; Raoul, J.-L.; Gourgou-Bourgade, S.; de la Fouchardière, C.; et al. FOLFIRINOX versus Gemcitabine for Metastatic Pancreatic Cancer. N. Engl. J. Med. 2011, 364, 1817-1825. [CrossRef] [PubMed]

4. Von Hoff, D.D.; Ervin, T.; Arena, F.P.; Chiorean, E.G.; Infante, J.; Moore, M.; Seay, T.; Tjulandin, S.A.; Ma, W.W.; Saleh, M.N.; et al. Increased Survival in Pancreatic Cancer with Nab-Paclitaxel plus Gemcitabine. N. Engl. J. Med. 2013, 369, 1691-1703. [CrossRef] [PubMed]

5. Corrigan, L.R.; Bracken-Clarke, D.M.; Horgan, A.M. The Challenge of Treating Older Patients with Pancreaticobiliary Malignancies. Curr. Probl. Cancer 2018, 42, 59-72. [CrossRef] [PubMed] 
6. van der Geest, L.G.M.; Haj Mohammad, N.; Besselink, M.G.H.; Lemmens, V.E.P.P.; Portielje, J.E.A.; van Laarhoven, H.W.M.; Wilmink, J.H.W. Nationwide Trends in Chemotherapy Use and Survival of Elderly Patients with Metastatic Pancreatic Cancer. Cancer Med. 2017, 6, 2840-2849. [CrossRef]

7. Ferrat, E.; Paillaud, E.; Laurent, M.; Le Thuaut, A.; Caillet, P.; Tournigand, C.; Lagrange, J.-L.; Canouï-Poitrine, F.; Bastuji-Garin, S.; ELPACA Study Group. Predictors of 1-Year Mortality in a Prospective Cohort of Elderly Patients with Cancer. J. Gerontol. A Biol. Sci. Med. Sci. 2015, 70, 1148-1155. [CrossRef]

8. Pamoukdjian, F.; Liuu, E.; Caillet, P.; Herbaud, S.; Gisselbrecht, M.; Poisson, J.; Boudou-Rouquette, P.; Zelek, L.; Paillaud, E. How to Optimize Cancer Treatment in Older Patients: An Overview of Available Geriatric Tools. Am. J. Clin. Oncol. 2019, 42, 109-116. [CrossRef]

9. von Elm, E.; Altman, D.G.; Egger, M.; Pocock, S.J.; Gøtzsche, P.C.; Vandenbroucke, J.P.; STROBE Initiative. The Strengthening the Reporting of Observational Studies in Epidemiology (STROBE) Statement: Guidelines for Reporting Observational Studies. PLoS Med. 2007, 4, e296. [CrossRef]

10. Barta, A. ICD-10-CM Official Coding Guidelines. J. AHIMA 2009, 80, 70-71.

11. Gnjidic, D.; Hilmer, S.N.; Blyth, F.M.; Naganathan, V.; Waite, L.; Seibel, M.J.; McLachlan, A.J.; Cumming, R.G.; Handelsman, D.J.; Le Couteur, D.G. Polypharmacy Cutoff and Outcomes: Five or More Medicines Were Used to Identify Community-Dwelling Older Men at Risk of Different Adverse Outcomes. J. Clin. Epidemiol. 2012, 65, 989-995. [CrossRef] [PubMed]

12. Katz, S.; Downs, T.D.; Cash, H.R.; Grotz, R.C. Progress in Development of the Index of ADL. Gerontologist 1970, 10, 20-30. [CrossRef] [PubMed]

13. Pamoukdjian, F.; Paillaud, E.; Zelek, L.; Laurent, M.; Lévy, V.; Landre, T.; Sebbane, G. Measurement of Gait Speed in Older Adults to Identify Complications Associated with Frailty: A Systematic Review. J. Geriatr. Oncol. 2015, 6, 484-496. [CrossRef] [PubMed]

14. Fried, L.P.; Tangen, C.M.; Walston, J.; Newman, A.B.; Hirsch, C.; Gottdiener, J.; Seeman, T.; Tracy, R.; Kop, W.J.; Burke, G.; et al. Frailty in Older Adults: Evidence for a Phenotype. J. Gerontol. Ser. A Biol. Sci. Med. Sci. 2001, 56, M146-M157. [CrossRef]

15. Raynaud-Simon, A.; Revel-Delhom, C.; Hébuterne, X. Clinical Practice Guidelines from the French Health High Authority: Nutritional Support Strategy in Protein-Energy Malnutrition in the Elderly. Clin. Nutr. 2011, 30, 312-319. [CrossRef]

16. Ai, Y.; Xu, R.; Liu, L. The Prevalence and Risk Factors of Sarcopenia in Patients with Type 2 Diabetes Mellitus: A Systematic Review and Meta-Analysis. Diabetol. Metab. Syndr. 2021, 13, 93. [CrossRef]

17. Montvida, O.; Shaw, J.; Atherton, J.J.; Stringer, F.; Paul, S.K. Long-Term Trends in Antidiabetes Drug Usage in the U.S.: Real-World Evidence in Patients Newly Diagnosed with Type 2 Diabetes. Diabetes Care 2018, 41, 69-78. [CrossRef]

18. Schernthaner, G.; Schernthaner, G.-H. The Right Place for Metformin Today. Diabetes Res. Clin. Pract. 2020, 159, 107946. [CrossRef]

19. Pamoukdjian, F.; Bouillet, T.; Lévy, V.; Soussan, M.; Zelek, L.; Paillaud, E. Prevalence and Predictive Value of Pre-Therapeutic Sarcopenia in Cancer Patients: A Systematic Review. Clin. Nutr. 2018, 37, 1101-1113. [CrossRef]

20. Broadhurst, P.J.; Hart, A.R. Metformin as an Adjunctive Therapy for Pancreatic Cancer: A Review of the Literature on Its Potential Therapeutic Use. Dig. Dis. Sci. 2018, 63, 2840-2852. [CrossRef]

21. Coyle, C.; Cafferty, F.H.; Vale, C.; Langley, R.E. Metformin as an Adjuvant Treatment for Cancer: A Systematic Review and Meta-Analysis. Ann. Oncol. 2016, 27, 2184-2195. [CrossRef] [PubMed]

22. Shi, Y.-Q.; Zhou, X.-C.; Du, P.; Yin, M.-Y.; Xu, L.; Chen, W.-J.; Xu, C.-F. Relationships Are between Metformin Use and Survival in Pancreatic Cancer Patients Concurrent with Diabetes: A systematic review and meta-analysis. Medicine 2020, 99 , e21687. [CrossRef] [PubMed]

23. Cetrone, M.; Mele, A.; Tricarico, D. Effects of the Antidiabetic Drugs on the Age-Related Atrophy and Sarcopenia Associated with Diabetes Type II. Curr. Diabetes Rev. 2014, 10, 231-237. [CrossRef] [PubMed]

24. Pamoukdjian, F.; Aparicio, T.; Zebachi, S.; Zelek, L.; Paillaud, E.; Canoui-Poitrine, F. Comparison of Mobility Indices for Predicting Early Death in Older Patients with Cancer: The Physical Frailty in Elderly Cancer Cohort Study. J. Gerontol. Ser. A 2020, 75, 189-196. [CrossRef] [PubMed]

25. Soubeyran, P.; Fonck, M.; Blanc-Bisson, C.; Blanc, J.-F.; Ceccaldi, J.; Mertens, C.; Imbert, Y.; Cany, L.; Vogt, L.; Dauba, J.; et al. Predictors of Early Death Risk in Older Patients Treated with First-Line Chemotherapy for Cancer. J. Clin. Oncol. 2012, 30, 1829-1834. [CrossRef]

26. Qi, Q.; Geng, Y.; Sun, M.; Wang, P.; Chen, Z. Clinical Implications of Systemic Inflammatory Response Markers as Independent Prognostic Factors for Advanced Pancreatic Cancer. Pancreatology 2015, 15, 145-150. [CrossRef]

27. Wu, L.; Zhu, L.; Xu, K.; Zhou, S.; Zhou, Y.; Zhang, T.; Hang, J.; Zee, B.C.-Y. Clinical Significance of Site-Specific Metastases in Pancreatic Cancer: A Study Based on Both Clinical Trial and Real-World Data. J. Cancer 2021, 12, 1715-1721. [CrossRef]

28. Pignon, F.; Turpin, A.; Hentic, O.; Coriat, R.; Salmon, E.; Baumgaertner, I.; Bertrand, N.; Lévy, P.; Rebours, V.; Hammel, P.; et al. Efficacy and Tolerance of Gemcitabine and Nab-Paclitaxel in Elderly Patients with Advanced Pancreatic Ductal Adenocarcinoma. Pancreatology 2021, 21, 1064-1070. [CrossRef]

29. Mizrahi, J.D.; Rogers, J.E.; Hess, K.R.; Wolff, R.A.; Varadhachary, G.R.; Javle, M.M.; Shroff, R.T.; Ho, L.; Fogelman, D.R.; Raghav, K.P.S.; et al. Modified FOLFIRINOX in Pancreatic Cancer Patients Age 75 or Older. Pancreatology 2020, 20, 501-504. [CrossRef]

30. Berger, A.K.; Haag, G.M.; Ehmann, M.; Byl, A.; Jäger, D.; Springfeld, C. Palliative Chemotherapy for Pancreatic Adenocarcinoma: A Retrospective Cohort Analysis of Efficacy and Toxicity of the FOLFIRINOX Regimen Focusing on the Older Patient. BMC Gastroenterol. 2017, 17, 143. [CrossRef] 
31. Costa, J.G.; de Jesus, V.H.F.; Camandaroba, M.P.G.; Dettino, A.L.A. Characteristics and Survival of Older Patients with Metastatic Pancreatic Cancer: A Retrospective Analysis of the AC Camargo Cancer Center Experience. Ther. Adv. Med. Oncol. 2019, 11, 1758835919874650. [CrossRef] [PubMed]

32. Hasegawa, R.; Okuwaki, K.; Kida, M.; Yamauchi, H.; Kawaguchi, Y.; Matsumoto, T.; Kaneko, T.; Miyata, E.; Uehara, K.; Iwai, T.; et al. A Clinical Trial to Assess the Feasibility and Efficacy of Nab-Paclitaxel plus Gemcitabine for Elderly Patients with Unresectable Advanced Pancreatic Cancer. Int. J. Clin. Oncol. 2019, 24, 1574-1581. [CrossRef] [PubMed]

33. Paillaud, E.; Soubeyran, P.; Caillet, P.; Cudennec, T.; Brain, E.; Terret, C.; Etchepare, F.; Mourey, L.; Aparicio, T.; Pamoukdjian, F.; et al. Multidisciplinary Development of the Geriatric Core Dataset for Clinical Research in Older Patients with Cancer: A French Initiative with International Survey. Eur. J. Cancer 2018, 103, 61-68. [CrossRef] [PubMed]

34. Angeli, E.; Chouahnia, K.; Canoui-Poitrine, F.; Duchemann, B.; Aparicio, T.; Paillaud, E.; Zelek, L.; Bousquet, G.; Pamoukdjian, F. Development, Validation and Clinical Impact of a Prediction Model for 6-Month Mortality in Older Cancer Patients: The GRADE. Aging 2020, 12, 4230-4246. [CrossRef] [PubMed] 